\title{
gs \\ Dependence of the Dresselhaus spin-orbit interaction on the quantum well width
}

\author{
M. P. Walser, ${ }^{1, *}$ U. Siegenthaler, ${ }^{1,2}$ V. Lechner, ${ }^{3}$ D. Schuh,${ }^{3}$ S. D. Ganichev, ${ }^{3}$ W. Wegscheider, ${ }^{4}$ and G. Salis ${ }^{1, \dagger}$ \\ ${ }^{1}$ IBM Research-Zurich, Säumerstrasse 4, CH-8803 Rüschlikon, Switzerland \\ ${ }^{2}$ Department of Physics, University of Basel, CH-4056 Basel, Switzerland \\ ${ }^{3}$ Terahertz Center, University of Regensburg, D-93053 Regensburg, Germany \\ ${ }^{4}$ Solid State Physics Laboratory, ETH Zurich, CH-8093 Zurich, Switzerland
}

(Received 3 October 2012; published 12 November 2012)

\begin{abstract}
We measured the Dresselhaus spin-orbit interaction coefficient $\beta_{1}$ for (001)-grown GaAs/ $\mathrm{Al}_{0.3} \mathrm{Ga}_{0.7} \mathrm{As}$ quantum wells for six different well widths $w$ between 6 and $30 \mathrm{~nm}$. The varying size quantization of the electron wave vector $z$-component $\left\langle k_{z}^{2}\right\rangle \sim(\pi / w)^{2}$ influences $\beta_{1}=-\gamma\left\langle k_{z}^{2}\right\rangle$ linearly. The value of the bulk Dresselhaus coefficient $\gamma=(-11 \pm 2) \mathrm{eV}^{3}$ was determined. We discuss the absolute sign of the Landé $g$ factors and the effective momentum scattering times.
\end{abstract}

DOI: 10.1103/PhysRevB.86.195309

PACS number(s): 71.70.Ej, 73.21.Fg, 72.25.Rb, 85.75.-d

Electron spins in semiconductor quantum structures experience spin-orbit interaction (SOI). For a two-dimensional electron gas (2DEG) hosted in a semiconductor quantum well (QW) with zinc-blende structure, there are two main sources for SOI, the Rashba ${ }^{1}$ and the Dresselhaus ${ }^{2,3}$ components. For spin-based applications, it is important to control the size of these two components. Of special interest is the situation with balanced Rashba and Dresselhaus SOI contributions, where the spin polarization of a helical spin state is preserved. ${ }^{4-8}$ Whereas Rashba SOI can be tuned and even eliminated either by using gate electrodes ${ }^{9,10}$ or by choosing a suitable ratio between the concentration of the modulation doping on the two sides of the QW, ${ }^{11}$ the Dresselhaus SOI is given by the choice of materials and the size quantization of the electron wave vector $\mathbf{k}$ along the growth direction $z$, that is, $\left\langle k_{z}^{2}\right\rangle=(\pi / w)^{2}$ for an infinitely high potential well of width $w$.

In this paper, we investigate experimentally the dependence of the Dresselhaus SOI on $w$ in $\mathrm{GaAs} / \mathrm{Al}_{0.3} \mathrm{Ga}_{0.7} \mathrm{As} \mathrm{QW}$ samples. The Dresselhaus SOI coefficient is measured using the technique described in Refs. 12 and 13, employing a small shift $\delta k \ll k_{\mathrm{F}}$ of the Fermi distribution in $\mathbf{k}$ space that leads to an average electron drift at the velocity $\hbar \delta \mathbf{k} / m$ (Fermi wave number $k_{\mathrm{F}}^{2}=2 \pi n_{\mathrm{s}}$, electron density $n_{\mathrm{s}}$, Planck constant $\hbar=h / 2 \pi$ ). This induces an average spin-orbit (SO) splitting $2 \beta^{*} \delta k$ of $\mathrm{QW}$ electrons in the conduction band that we determine from a change in the spin precession frequency $\nu$. The coefficient $\beta^{*}=\beta_{1}-2 \beta_{3}$ contains both linear $\left(\beta_{1}=-\gamma\left\langle k_{z}^{2}\right\rangle\right)$ and cubic $\left(\beta_{3}=-\frac{1}{4} \gamma k_{\mathrm{F}}^{2}\right)$ Dresselhaus terms, which are both proportional to the bulk Dresselhaus coefficient $\gamma$. Rashba SOI contributions $\alpha \ll \beta_{1}$ play a minor role in this study.

We measured six similarly grown (001)-orientated GaAs $/ \mathrm{Al}_{0.3} \mathrm{Ga}_{0.7} \mathrm{As}$ structures, each hosting 15 equivalent Si- $\delta$-doped QWs ${ }^{14}$ with $w=6-30 \mathrm{~nm}$, and obtained $\gamma=$ $(-11 \pm 2) \mathrm{eV} \AA^{3}$ from a linear fit to $\beta_{1}=-\gamma\left\langle k_{z}^{2}\right\rangle$. Despite many investigations of a wide variety of GaAs-based structures, the precise value of $\gamma$ is still being discussed controversially in literature. Various experimental techniques claim $\gamma$ values between 3 and $35 \mathrm{eV} \AA^{3}$, and most theoretical calculations ${ }^{15-19}$ exhibit relatively poor agreement with experimental data. ${ }^{16,19-23}$

To measure $v$, we use time-resolved Kerr rotation (TRKR). For this, circularly polarized Ti:sapphire laser pulses at a repetition rate of $80 \mathrm{MHz}$ generate a small spin polarization $\mathbf{S}\|z\|[001]$ in the conduction band of the QW. The time evolution of $S_{z}$ is then monitored in an external magnetic field $\mathbf{B}_{\text {ext }} \|$ [100] using TRKR at a base temperature of $20 \mathrm{~K}$. Traces of $S_{z}$ vs time $t$ are fit to an exponentially decaying cosine function $\propto \exp \left(-t / \tau_{s}\right) \cos (2 \pi v t)$ to determine $v$ and the spin lifetime $\tau_{s}$. Nonoscillating signals are fit to a single exponential. The decay time for $\mathbf{S} \|[001]$ and $B_{\text {ext }}=0$ is denoted by $\tau_{z}$. For the 6-, 8-, and 30-nm QW, we used two frequencies to fit the data, one for the $\mathrm{QW}$ spins and one for the bulk GaAs spins, and selected the $v$ and $\tau_{s}$ that belong to the QW spins. Mesalike channels of width $W=100 \mu \mathrm{m}$ are etched into the substrates to apply an electric current $\mathbf{I} \|[100]$. We assume an equal $n_{\mathrm{s}}$ and current density $\mathbf{j}_{\mathrm{s}}=\mathbf{I} /(15 \mathrm{~W})$ per $\mathrm{QW}$ and estimate $\hbar \delta \mathbf{k} / m=\mathbf{j}_{\mathrm{s}} / e n_{\mathrm{s}}$ (electron charge $e$, effective mass $m=0.067 m_{0}$, free electron mass $m_{0}$ ). The energy per area of the pump pulses was kept below $4 \times 10^{-3} \mathrm{Jm}^{-2}$ to ensure that the number of excited electron-hole pairs is much smaller than $n_{\mathrm{s}}$. The mobility $\mu$ and $n_{\mathrm{s}}$ were measured separately by Hall measurements.

The measured $h v$ is influenced by an average SO splitting $\left\langle E_{\mathrm{D}}\right\rangle=2 \beta^{*} \delta k$ and the Zeeman splitting $E_{\mathrm{Z}}=g \mu_{\mathrm{B}} B_{\text {ext }}$ (Ref. 13). Here, $g$ is the electron Lande factor, and $\mu_{\mathrm{B}}$ is Bohr's magneton. Because of the chosen orientation of $\mathbf{B}_{\mathrm{ext}}$ and $\mathbf{I}$, the two energies simply add, and

$$
h v=\left\langle E_{\mathrm{D}}\right\rangle+E_{\mathrm{Z}} \text {. }
$$

We determine the modulus of $g$ from the precession frequency $v_{0}$ measured at $\delta k=0$. There, $\left\langle E_{\mathrm{D}}\right\rangle=0$, and we can use $g=h v_{0} /\left(\mu_{\mathrm{B}} B_{\text {ext }}\right)$. Note that the signs of $g$ and $h v$ cannot be found from TRKR measurements directly, but follow from a comparison of $\left\langle E_{\mathrm{D}}\right\rangle$ and $E_{\mathrm{Z}}$, as we will discuss below. When $\delta k$ is varied, $v$ changes linearly, and $\beta^{*}$ follows from the slope $2 \beta^{*}=\partial(h v) / \partial \delta k$ at $\delta k=0$.

Figure 1(a) shows traces of $S_{z}(t)$ for a 8 -nm-wide QW (labels 1-4). Because $\beta_{1}$ is relatively large in this sample, spins decay quickly. Spin precession is very slow at $\delta k=0$ (label 2), indicating a small $g$ factor. The upper inset shows the fitted $|v|$ vs $\delta k$. Spins precess faster at $\delta k=-8.8 \times 10^{6} \mathrm{~m}^{-1}$ (label 1) than for the opposite sign of $\delta k$ (label 4). From this and Eq. (1), it can be concluded that $\left\langle E_{\mathrm{D}}\right\rangle$ and $E_{\mathrm{Z}}$ have opposite signs for positive $\delta k$. This is in agreement with the observation of $h v=0$ for a positive $\delta k \approx 2.6 \times 10^{6} \mathrm{~m}^{-1}$ (label 3), which 

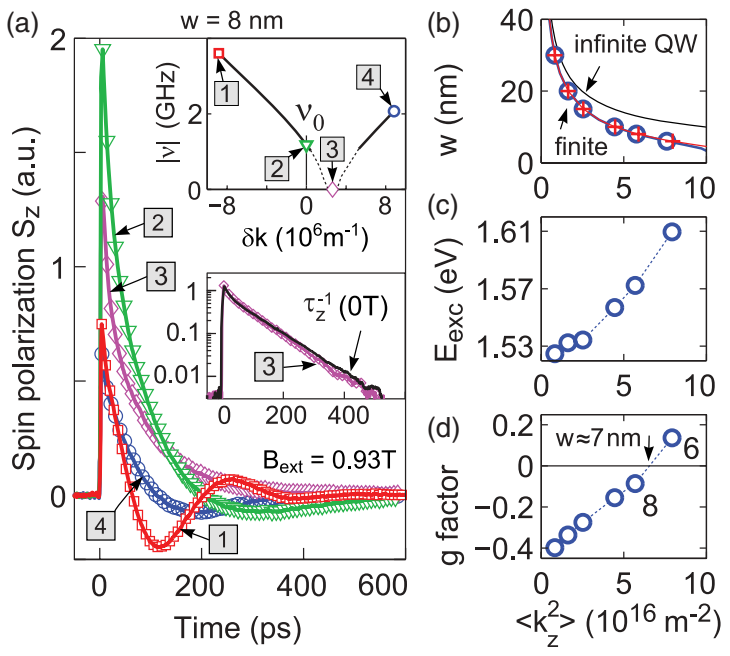

FIG. 1. (Color online) (a) Measured $S_{z}(t)$ for $w=8 \mathrm{~nm}$ at $B_{\text {ext }}=$ $0.93 \mathrm{~T}$ and various $\delta k$ (labels $1-4$ ). (Upper inset) The fitted spin precession frequency $v$ strongly varies with $\delta k$. The regime of $|v|<$ $1 \mathrm{GHz}$ (dotted line) is not accessible because of the fast signal decay. (Lower inset) No oscillation is observed for this signal (label 3); it decays exponentially with $\tau_{s} \approx \tau_{z}$. (b) Variation of $\left\langle k_{z}^{2}\right\rangle$ with $w$ for an infinite and a finite $\mathrm{GaAs} / \mathrm{Al}_{0.3} \mathrm{Ga}_{0.7}$ As square potential well. Shown are analytically (circles) and numerically $(+)$ calculated values for $n_{\mathrm{s}}=0$ and $1.2 \times 10^{15} \mathrm{~m}^{-2}$. (c) Kerr excitation energy $E_{\text {exc }}$ and (d) $g$ factor vs $\left\langle k_{z}^{2}\right\rangle$. Note the sign change of $g$ at $w \approx 7 \mathrm{~nm}$ (arrow).

is explained by a cancellation of the two energies. At this point, we find an exponential spin decay with $\tau_{s} \approx \tau_{z}$ (lower inset). From this follows that $\beta^{*}$ and $g$ must have opposite signs in this sample.

Different QW widths $w$ result in different values for $\left\langle k_{z}^{2}\right\rangle$. Figure 1(b) shows $\left\langle k_{z}^{2}\right\rangle=(\pi / w)^{2}$, the result for an infinitely high potential well. In comparison, numerical ${ }^{24}$ or analytical ${ }^{25}$ calculations for realistic QWs with a conduction-band offset of $0.262 \mathrm{eV}$ between the well and the barrier region yield substantially smaller values, because of wave-function leakage into the barrier regions. Note that also the approximation $\left\langle k_{z}^{2}\right\rangle=2 m E_{0} / \hbar^{2}$ with the subband energy $E_{0}$ yields too large values for $\left\langle k_{z}^{2}\right\rangle$ because of the finite contribution from the barrier potential energy. In the following, we will use the numerically obtained values of $\left\langle k_{z}^{2}\right\rangle$ to discuss our data. This affects the $\gamma$ values obtained from $\beta_{1}$. For example, for a 6-nm QW, $\beta_{1} /\left\langle k_{z}^{2}\right\rangle$ is 3.5 times less if $\left\langle k_{z}^{2}\right\rangle=(\pi / w)^{2}$ is used, and 1.5 times less if $\left\langle k_{z}^{2}\right\rangle=2 m E_{0} / \hbar^{2}$ is used. The application of the latter approximation in Refs. 21 and 23 could explain the postulated dependence of $\gamma$ on the QW width $w$.

We chose a laser excitation energy $E_{\text {exc }}$ close to the QW absorbtion edge, where the TRKR signal is maximum. $E_{\mathrm{exc}}$ is a measure for the interband transition energy between quantized valence- and conduction-band states. For $w=30 \mathrm{~nm}, E_{\mathrm{exc}}$ is approximately the bulk GaAs bandgap $(1.52 \mathrm{eV}$ at $20 \mathrm{~K})$, and then increases with stronger confinement [Fig. 1(c)]. In Fig. 1(d), we show the $g$ factors obtained in this study as a function of $\left\langle k_{z}^{2}\right\rangle$. From theory and experiments, ${ }^{26-29}$ we expect $g$ to change sign between $w=6$ and $8 \mathrm{~nm}$. Here, the sign change at $w \approx 7 \mathrm{~nm}$ is not a priori assumed but directly observed, as explained below.

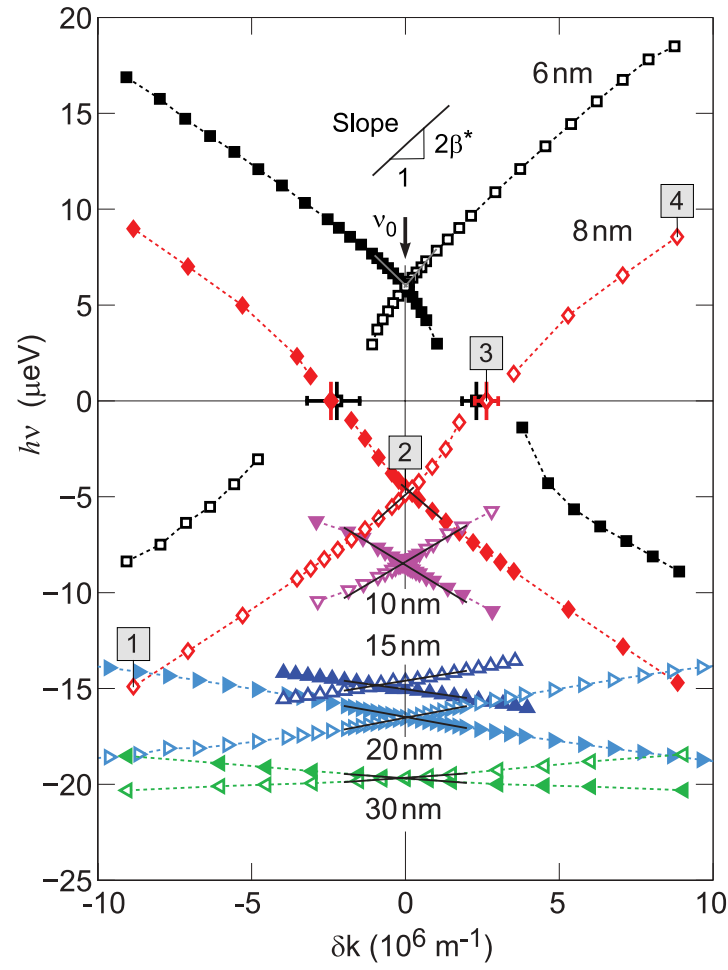

FIG. 2. (Color online) Measured Zeeman and drift-induced Dresselhaus SO splitting $h v=g \mu_{\mathrm{B}} B_{\mathrm{ext}}+2 \beta^{*} \delta k$ as a function of $\delta k$ for 6to 30-nm-wide QWs and for $B_{\text {ext }}>0$ (open symbols). For $B_{\text {ext }}<0$, we plot $-h v$ (solid symbols). The Zeeman splitting $g \mu_{\mathrm{B}} B_{\text {ext }}$ changes sign between $w=6$ and $8 \mathrm{~nm}$. $2 \beta^{*}$ is obtained from the slopes $\partial(h v) / \partial \delta k$ in the regime of $|\delta k|<2 \times 10^{6} \mathrm{~m}^{-1}$ (solid lines in the vicinity of $\delta k=0$ ). For $w=6$ and $8 \mathrm{~nm}$, we used the regime of $|v|>\left|v_{0}\right|$ and $|\delta k|<1 \times 10^{6} \mathrm{~m}^{-1}$.

In Fig. 2 all measured spin splittings $h v$ are plotted as a function of $\delta k$. We choose the sign in consistency with Eq. (1) by assuming that $\beta^{*}$ has the same sign for all QWs and that $g$ is negative for wide QWs. We plot $+h v$ for $B_{\mathrm{ext}}>0$ and $-h v$ for $B_{\text {ext }}<0$. This way the two curves intercept at positive values for $g>0$ and negative values for $g<0$. $B_{\text {ext }}$ was set to $\pm 0.85 \mathrm{~T}$ for the 6-, 20-, and 30-nm QWs and to $\pm 0.93 \mathrm{~T}$ for the 8-, 10-, and 15-nm QWs.

To determine the relative signs of $\beta^{*}$ and $g$, we analyzed the TRKR data of all QWs in the same way as for the 8-nm QW discussed above. For all but the 6-nm QW, $\beta^{*}$ and $g$ have opposite signs. From this, we directly conclude that $g>0$ for $w=6 \mathrm{~nm}, g<0$ for $w>8 \mathrm{~nm}$, and $\beta^{*}>0$ for all samples. Note that $\beta^{*}$ could possibly change sign with increasing $w$ if $2 \beta_{3}>\beta_{1}$. However, even for $w=30 \mathrm{~nm}$, this is not the case here, because $\beta_{3} \approx 3 \times 10^{-14} \mathrm{eVm}$ is sufficiently small.

We obtain $\beta^{*}$ individually for each sample from the slopes of $v$ versus $\delta k$ in Fig. 2. To calculate the linear Dresselhaus term $\beta_{1}=\beta^{*}+2 \beta_{3}$, the cubic correction $\beta_{3}=-\frac{1}{4} \gamma k_{\mathrm{F}}^{2}$ and therefore $\gamma$ needs to be known ( $k_{\mathrm{F}}$ is calculated from the measured $\left.n_{\mathrm{s}}\right)$. We find a single value of $\gamma=(-11 \pm 2) \mathrm{eV} \AA^{3}$ for all samples in a self-consistent loop where first $\beta_{3}$ is calculated and then the slope of $\beta_{1}$ versus $\left\langle k_{z}^{2}\right\rangle$ is fitted [Fig. 3(a)] to iteratively adapt $\gamma$. A list of all SOI coefficients is given in Table I. The obtained value of $\gamma$ is more precise than 


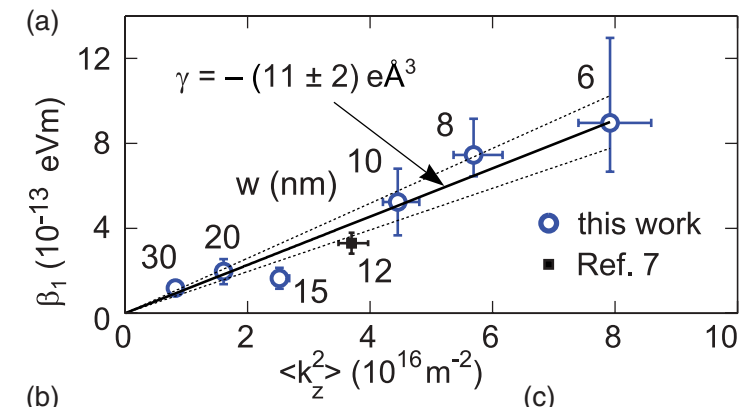

(b)

(c)
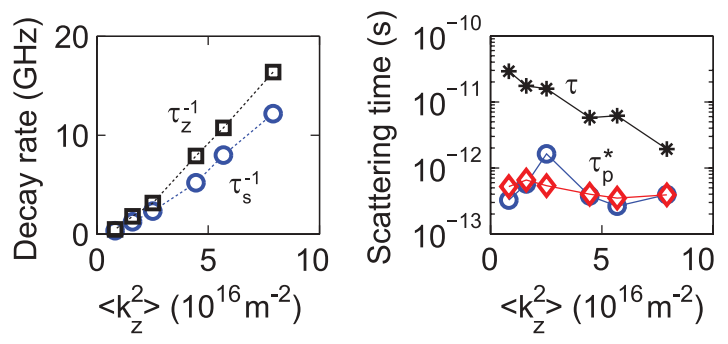

FIG. 3. (Color online) (a) Measured $\beta_{1}$ (open symbols) and data from Ref. 7 (solid symbol) vs $\left\langle k_{z}^{2}\right\rangle$ are fit to $-\gamma\left\langle k_{z}^{2}\right\rangle$ (solid line). (Dotted lines) 95\% confidence interval. (Horizontal error bars) $\pm 0.5 \mathrm{~nm}$ variation in $w$. (Vertical error bar) $\pm 30 \%$ variation in $n_{\mathrm{s}}$ and estimated uncertainty in the fitted $\nu$. (b) Spin decay rates $\tau_{s}^{-1}$ (circles) and $\tau_{z}^{-1}$ (squares) vs $\left\langle k_{z}^{2}\right\rangle$. (c) Comparison of $\tau$ and $\tau_{p}^{*} ; \tau$ was calculated from the mobility $\mu, \tau_{p}^{*}$ was calculated from $\tau_{s}, \beta_{1}$ and $k_{\mathrm{F}}^{2}$ (circles), and from $\tau_{s},-\gamma\left\langle k_{z}^{2}\right\rangle$, and $k_{\mathrm{F}}^{2}$ (diamonds).

in a previous experiment with only one $\mathrm{QW}$ width ${ }^{13}$ and agrees with other experimental ${ }^{19-21,23}$ and theoretical ${ }^{15,16,18}$ work.

The linear fit shown in Fig. 3(a) describes both wide (20$30 \mathrm{~nm})$ and narrow $(6-8 \mathrm{~nm})$ QWs with a single $\gamma$ reasonably. The error bars for $w=10,15,20$, and $30 \mathrm{~nm}$ follow from an estimated uncertainty in $n_{\mathrm{s}}$ of $\pm 30 \%$. For $w=6$ and $8 \mathrm{~nm}$, we consider the low confidence level for the fit of $v$ to be the dominant error source. In particular for $|v|<\left|v_{0}\right|$, TRKR data spans less than a full precession period. Therefore the data for $|v|>\left|v_{0}\right|$ is used to determine $\beta^{*}$, and then an upper limit for $\beta^{*}$ is estimated from the regime of $|v|<\left|v_{0}\right|$, and a lower limit from the regime of $|v| \gg\left|v_{0}\right|$.

Compared with measurements on one single QW width, the $\gamma$ obtained here is less sensitive to systematic errors caused by peculiarities of individual samples. For $w=15 \mathrm{~nm}$, the measured $\beta_{1}$ deviates the most from $-\gamma\left\langle k_{z}^{2}\right\rangle$. It is unlikely that this QW has been grown with an accidentally larger $w$, because $E_{\text {exc }}, g$, and the decay rates vary rather monotonously with $\left\langle k_{z}^{2}\right\rangle$. Local screening of the in-plane electric field by optically generated charge carriers could possibly reduce $\delta k$, but we do not find changes in $\beta^{*}$ of more than $20 \%$ when decreasing the pump power. Most probably an unequal current distribution among the 15 QWs in this sample leads to an actual drift in the relevant QWs that is smaller than anticipated from the measured $\mathbf{I}$, and this could explain the lower $\beta_{1}$ value. We note that all samples in this study have been grown in the same week at very similar conditions, except the 15-nm QW, which was grown earlier and processed slightly differently.

Previously we have studied SOI for a strongly asymmetric 12-nm-wide single QW by analysis of spatially resolved spin polarization maps. ${ }^{7}$ To support our findings, data from this reference is also shown in Fig. 3(a) and has been included in the fit of $\gamma$. The 15- and 12-nm QWs suggest a slightly lower $\gamma$ than discussed above, and a fit to the data for $w \geqslant 15 \mathrm{~nm}$ yields $\gamma \approx-9 \mathrm{eV} \AA^{3}$.

Finally, we discuss the spin decay rates and determine the effective momentum scattering time $\tau_{p}^{*}$, which is typically difficult to estimate from the transport scattering time $\tau=$ $\mu m / e$. In general, $\tau_{p}^{*}$ is smaller than $\tau$ (Refs. 30 and 31 ), because $\tau$ is rather insensitive to forward scattering and is not affected by electron-electron scattering. We expect spin lifetime to be limited by the Dyakonov Perel (DP) mechanism. ${ }^{32}$ Fast scattering, that is, $E_{\mathrm{D}} / h \ll 1 / \tau_{p}^{*}$, will result in a random walk of $\mathbf{S}$ on the Bloch sphere, and for strong SOI, $S_{z}$ will decay quickly. For $\alpha \ll \beta_{1}$ and $\beta_{3} \ll \beta_{1}$, the DP decay rates are $1 / \tau_{s}=3 \beta_{1}^{2} k_{\mathrm{F}}^{2} \tau_{p}^{*} / \hbar^{2}$ and $1 / \tau_{z}=4 \beta_{1}^{2} k_{\mathrm{F}}^{2} \tau_{p}^{*} / \hbar^{2}$ (Refs. 33 and 34). From these rates it is possible to determine $\tau_{p}^{*}$ if $\beta_{1}$ is known. ${ }^{21}$

In Fig. 3(b) we show $1 / \tau_{s}$ and $1 / \tau_{z}$ vs $\left\langle k_{z}^{2}\right\rangle$. We do not observe a quadratic increase of these rates with $\beta_{1}$, which indicates that either $\tau_{p}^{*}$ or $k_{\mathrm{F}}^{2}$ depends on $w$ (Ref. 35). Since the decay rates, $\beta_{1}$ and $k_{\mathrm{F}}^{2}$ were measured independently, we can determine $\tau_{p}^{*}$, as shown in Fig. 3(c) (circles). We find $\tau_{p}^{*}$ to be one to two orders of magnitude smaller than $\tau$. Because the individually measured $\beta_{1}$ values do not increase perfectly linearly with $\left\langle k_{z}^{2}\right\rangle$, we also used linearly interpolated values for $\beta_{1}=-\gamma\left\langle k_{z}^{2}\right\rangle$. This yields $\tau_{p}^{*} \approx 0.5$ ps for all $w$ (diamonds). The measured $\beta_{1}$ for $w=15 \mathrm{~nm}$ yields a substantially larger $\tau_{p}^{*}$, which suggests that for this sample the measured $\beta_{1}$ is too small.

For a 2DEG closely below the transition from a degenerate to nondegenerate statistics, electron-electron scattering is expected to be the predominant mechanism contributing to $\tau_{p}^{*}$ (Refs. 25, 30, and 36), whereas interface scattering is expected

TABLE I. List of QWs and parameters as follows: $w$, nominal QW width; $\left\langle k_{z}^{2}\right\rangle$ numerically calculated; $n_{\mathrm{s}}$ and $\mu$ from Hall measurements; $\tau$ from $\mu ; g$ and $\beta^{*}$ are determined from $h v$ vs $\delta k ; \beta_{1}$ and $\beta_{3}$ are calculated from $\beta^{*}, \gamma$, and $k_{\mathrm{F}}^{2} ; \tau_{p}^{*}$ is calculated from $\tau_{s}, \gamma$, and $\left\langle k_{z}^{2}\right\rangle$.

\begin{tabular}{|c|c|c|c|c|c|c|c|c|c|c|}
\hline \multirow{2}{*}{$\begin{array}{l}w \\
\mathrm{~nm}\end{array}$} & \multirow{2}{*}{$\begin{array}{l}g \\
-\end{array}$} & \multirow{2}{*}{$\begin{array}{c}n_{\mathrm{s}} \\
10^{15} \mathrm{~m}^{-2}\end{array}$} & \multirow{2}{*}{$\begin{array}{c}\mu \\
\mathrm{m}^{2} / \mathrm{Vs}\end{array}$} & $\left\langle k_{z}^{2}\right\rangle$ & $k_{\mathrm{F}}^{2}$ & $\beta^{*}$ & $\beta_{1}$ & $2 \beta_{3}$ & $\tau$ & $\tau_{p}^{*}$ \\
\hline & & & & \multicolumn{2}{|c|}{$10^{16} \mathrm{~m}^{-2}$} & \multicolumn{3}{|c|}{$10^{-13} \mathrm{eVm}$} & \multicolumn{2}{|c|}{ ps } \\
\hline 6 & 0.14 & 0.9 & 5 & 7.9 & 0.6 & 8.7 & 9.0 & 0.3 & 2 & 0.4 \\
\hline 8 & -0.09 & 1.3 & 16 & 5.7 & 0.8 & 7.0 & 7.4 & 0.4 & 6 & 0.3 \\
\hline 10 & -0.16 & 1.2 & 15 & 4.5 & 0.7 & 4.8 & 5.2 & 0.4 & 6 & 0.4 \\
\hline 15 & -0.28 & 1.2 & 41 & 2.5 & 0.8 & 1.2 & 1.6 & 0.4 & 16 & 0.5 \\
\hline 20 & -0.34 & 1.3 & 46 & 1.6 & 0.8 & 1.5 & 2.0 & 0.5 & 18 & 0.6 \\
\hline 30 & -0.40 & 1.7 & 76 & 0.8 & 1.1 & 0.6 & 1.2 & 0.6 & 29 & 0.5 \\
\hline
\end{tabular}


to contribute significantly to $\tau$, in particular for small $w$. For a degenerate 2DEG, $1 / \tau_{e-e} \approx \frac{\pi}{2}\left(E_{\mathrm{F}} / \hbar\right) \times\left(k_{\mathrm{B}} T / E_{\mathrm{F}}\right)^{2} \times$ $\ln \left(E_{\mathrm{F}} / k_{\mathrm{B}} T\right)$ (Refs. 37 and 31$)$. Using $E_{\mathrm{F}} \approx 4.5 \mathrm{meV}$ and $T=$ $20 \mathrm{~K}$ we estimate $\tau_{e-e} \approx 0.55 \mathrm{ps}$. Assuming $1 / \tau_{e-e}=1 / \tau_{p}^{*}-$ $1 / \tau$, we determine $\tau_{e-e} \approx 0.5 \mathrm{ps}$ from the measurement, which agrees with the theoretical expectation ${ }^{37}$ and other experimental work. ${ }^{30}$ This also explains why $\tau_{p}^{*}$ is essentially $w$ independent, despite the strong decrease of $\tau$ with decreasing $w$.

In summary, we have measured the Dresselhaus SOI in $\mathrm{GaAs} / \mathrm{Al}_{0.3} \mathrm{Ga}_{0.7} \mathrm{As} \mathrm{QWs}$ of different widths $w$ and found a linear increase with the confinement parameter $\left\langle k_{z}^{2}\right\rangle$. From this we determined the bulk Dresselhaus coefficient $\gamma=$ $(-11 \pm 2) \mathrm{eV} \AA^{3}$. The sign of the $g$ factors was measured and the sign change at $w \approx 7 \mathrm{~nm}$ was observed. Relating the measured DP spin lifetimes to the measured spin-orbit splittings, the effective momentum scattering time $\tau_{p}^{*} \approx 0.5 \mathrm{ps}$ was determined, stressing the importance of electron-electron scattering for the dynamics of spin.

We acknowledge financial support from the Swiss National Science Foundation through National Center of Competence in Research (NCCR) Nano and NCCR QSIT. We thank R. Allenspach, K. Ensslin, M. Poggio, and Y. S. Chen for discussions and M. Tschudy, U. Drechsler, and S. Reidt for technical support. S.D.G. and V.L. thank DFG for support via Program No. SPP 1285. *wal@zurich.ibm.com

†gsa@zurich.ibm.com

${ }^{1}$ Y. A. Bychkov and E. I. Rashba, J. Phys. C 17, 6039 (1984).

${ }^{2}$ G. Dresselhaus, Phys. Rev. 100, 580 (1955).

${ }^{3}$ M. I. D'yakonov and V. Yu. Kachorovskii, Fiz. Tekh. Poluprovodn.

20, 178 (1986) [Sov. Phys. Semicond. 20, 110 (1986)].

${ }^{4}$ J. Schliemann, J. C. Egues, and D. Loss, Phys. Rev. Lett. 90, 146801 (2003).

${ }^{5}$ B. A. Bernevig, J. Orenstein, and S.-C. Zhang, Phys. Rev. Lett. 97, 236601 (2006).

${ }^{6}$ J. D. Koralek, C. P. Weber, J. Orenstein, B. A. Bernevig, S.-C. Zhang, S. Mack, and D. D. Awschalom, Nature (London) 458, 610 (2009).

${ }^{7}$ M. P. Walser, C. Reichl, W. Wegscheider, and G. Salis, Nat. Phys. 8, 757 (2012).

${ }^{8}$ M. Kohda, V. Lechner, Y. Kunihashi, T. Dollinger, P. Olbrich, C. Schönhuber, I. Caspers, V. V. Bel'kov, L. E. Golub, D. Weiss, K. Richter, J. Nitta, and S. D. Ganichev, Phys. Rev. B 86, 081306 (2012).

${ }^{9}$ J. Nitta, T. Akazaki, H. Takayanagi, and T. Enoki, Phys. Rev. Lett. 78, 1335 (1997).

${ }^{10}$ M. Studer, G. Salis, K. Ensslin, D. C. Driscoll, and A. C. Gossard, Phys. Rev. Lett. 103, 027201 (2009).

${ }^{11}$ V. Lechner, L. E. Golub, P. Olbrich, S. Stachel, D. Schuh, W. Wegscheider, V. V. Bel'kov, and S. D. Ganichev, Appl. Phys. Lett. 94, 242109 (2009).

${ }^{12}$ L. Meier, G. Salis, I. Shorubalko, E. Gini, S. Schön, and K. Ensslin, Nat. Phys. 3, 650 (2007).

${ }^{13}$ M. Studer, M. P. Walser, S. Baer, H. Rusterholz, S. Schön, D. Schuh, W. Wegscheider, K. Ensslin, and G. Salis, Phys. Rev. B 82, 235320 (2010).

${ }^{14}$ V. Lechner, L. E. Golub, F. Lomakina, V. V. Bel'kov, P. Olbrich, S. Stachel, I. Caspers, M. Griesbeck, M. Kugler, M. J. Hirmer, T. Korn, C. Schüller, D. Schuh, W. Wegscheider, and S. D. Ganichev, Phys. Rev. B 83, 155313 (2011).

${ }^{15}$ P. V. Santos and M. Cardona, Phys. Rev. Lett. 72, 432 (1994).

${ }^{16}$ W. Knap, C. Skierbiszewski, A. Zduniak, E. Litwin-Staszewska, D. Bertho, F. Kobbi, J. L. Robert, G. E. Pikus, F. G. Pikus, S. V. Iordanskii, V. Mosser, K. Zekentes, and Y. B. Lyanda-Geller, Phys. Rev. B 53, 3912 (1996).

${ }^{17}$ R. Winkler, Spin-Orbit Coupling Effects in Two-Dimensional Electron and Hole Systems (Springer, Berlin, 2003).

${ }^{18}$ A. N. Chantis, M. van Schilfgaarde, and T. Kotani, Phys. Rev. Lett. 96, 086405 (2006).
${ }^{19}$ J. J. Krich and B. I. Halperin, Phys. Rev. Lett. 98, 226802 (2007); see also supplementary information therein.

${ }^{20}$ D. Richards, B. Jusserand, G. Allan, C. Priester, and B. Etienne, Solid-State Electron. 40, 127 (1996).

${ }^{21}$ W. J. H. Leyland, R. T. Harley, M. Henini, A. J. Shields, I. Farrer, and D. A. Ritchie, Phys. Rev. B 76, 195305 (2007).

${ }^{22}$ S. Faniel, T. Matsuura, S. Mineshige, Y. Sekine, and T. Koga, Phys. Rev. B 83, 115309 (2011).

${ }^{23}$ P. S. Eldridge, J. Hübner, S. Oertel, R. T. Harley, M. Henini, and M. Oestreich, Phys. Rev. B 83, 041301 (2011).

${ }^{24}$ Calculations were done with the NEXTNANO3 software, Version 2004-Aug-24. We calculated the envelope function $\psi(z)$ of the QW ground state and used $\left|k_{z}^{2}\right|:=\int\left|\psi^{\prime}(z)\right|^{2} d z$.

${ }^{25}$ Optical Spectroscopy of Semicondctor Nanostructures, edited by E. L. Ivchenko (Alpha Science Interantional, Harrow, 2005).

${ }^{26}$ M. J. Snelling, G. P. Flinn, A. S. Plaut, R. T. Harley, A. C. Tropper, R. Eccleston, and C. C. Phillips, Phys. Rev. B 44, 11345 (1991).

${ }^{27}$ E. Ivchenko, A. Kiselev, and M. Willander, Solid State Commun. 102, 375 (1997).

${ }^{28}$ P. L. Jeuney, D. Robarty, X. Mariey, T. Amandy, M. Brousseauy, J. Barrauy, V. Kalevichz, and D. Rodichevx, Semicond. Sci. Technol. 12, 380 (1997)

${ }^{29}$ I. A. Yugova, A. Greilich, D. R. Yakovlev, A. A. Kiselev, M. Bayer, V. V. Petrov, Y. K. Dolgikh, D. Reuter, and A. D. Wieck, Phys. Rev. B 75, 245302 (2007).

${ }^{30}$ M. A. Brand, A. Malinowski, O. Z. Karimov, P. A. Marsden, R. T. Harley, A. J. Shields, D. Sanvitto, D. A. Ritchie, and M. Y. Simmons, Phys. Rev. Lett. 89, 236601 (2002).

${ }^{31}$ M. Glazov and E. Ivchenko, J. Exp. Theor. Phys. 99, 1279 (2004).

${ }^{32}$ M. I. D'yakonov and V. I. Perel', Sov. Phys. Solid State 13, 3023 (1972).

${ }^{33}$ N. S. Averkiev, L. E. Golub, and M. Willander, J. Phys.: Condens. Matter 14, R271 (2002).

${ }^{34}$ J. Kainz, U. Rössler, and R. Winkler, Phys. Rev. B 70, 195322 (2004).

${ }^{35}$ R. S. Britton, T. Grevatt, A. Malinowski, R. T. Harley, P. Perozzo, A. R. Cameron, and A. Miller, Appl. Phys. Lett. 73, 2140 (1998).

${ }^{36}$ W. J. H. Leyland, G. H. John, R. T. Harley, M. M. Glazov, E. L. Ivchenko, D. A. Ritchie, I. Farrer, A. J. Shields, and M. Henini, Phys. Rev. B 75, 165309 (2007).

${ }^{37}$ H. Fukuyama and E. Abrahams, Phys. Rev. B 27, 5976 (1983). 